\title{
Facile synthesis of arylsulfonates from phenol derivatives and sulfonyl chlorides
}

\author{
Felix N. Ngassa*, Sean Riley, Tsvetelina P. Atanasova, Abdurahman O. Ahmed, Stacey Kerr, \\ Tyler A. Cooley, Ibrahim Al Shaikh Dawood, Ethan R. Austhof, Jonah R. J. Duran and \\ Mitch Franklin \\ Department of Chemistry, Grand Valley State University, 1 Campus Drive, Allendale, MI 49401, USA.
}

\begin{abstract}
The C-O bond of phenol can be activated by reaction with arylsulfonyl chlorides to yield the corresponding arylsulfonates that can be used as electrophilic partners in subsequent reactions. Arylsulfonates can be conveniently synthesized from inexpensive phenol derivatives and sulfonyl chlorides. Moreover, their crystalline nature makes arylsulfonates easier than other electrophiles to purify and store over a long period of time. Although arylsulfonates are inferior to triflates in terms of electrophilicity, triflates are better leaving groups compared to sulfonates; arylsulfonates however have greater economical advantages over triflates. Facile synthesis of different arylsulfonates from various arylsulfonyl chlorides and phenol are reported. What is unique about the synthesis is that very good to excellent yields of arylsulfonates were obtained without the use of column chromatography. A more efficient purification technique was developed based on recrystallization. Using 2,4-dinitrophenyl 4-methylbenzenesulfonate and various amines, initial experiments have shown greater regioselectivity for the $S_{N} A r$ products.
\end{abstract}

KEYWORDS: arylsulfonates, electrophiles, phenol, nucleophilic aromatic substitution, synthesis

\section{INTRODUCTION}

Organosulfur compounds are commonly found in many synthetic drugs, in nature and many biological

\footnotetext{
*Corresponding author: ngassaf@gvsu.edu
}

systems [1]. The sulfonate ester moiety is a potent electrophile that is used in several important transformations in organic synthesis. Sulfonate esters can be formed through typical esterification of sulfonic acids, and through transesterifications involving an alcohol and sulfonate ester. Alternatively, sulfonate esters may be formed via the highly efficient nucleophilic acyl substitution reaction of alcohols and sulfonyl halides [2-7]. The efficiency of these reactions has led to the widespread use of sulfonate formation as a means to protect alcohols in the course of multistep syntheses [8]. Sulfonate esters may also be used as substrates in various synthetic transformations. This protecting group/substrate duality allows for protection and subsequent transformation, which can be very practical in multistep syntheses. Under mild acidic conditions, most sulfonates are generally stable. The sulfonate ester group is intrinsically labile [9]. For example it has been demonstrated that $\mathrm{NaOH}$ or $\mathrm{BBr}_{3}$ can cleave most sulfonate ester groups under nonaqueous conditions [9].

Synthetically, sulfonate esters are characterized as good leaving groups, trumping the reactivity of the common halide leaving groups, like chlorine and bromine. Thus, sulfonate esters can be similarly applied in substitution, elimination, reduction, and transition metal-catalyzed reactions [10-13]. The possibility to use sulfonates as alternative substrates to halides is advantageous in that it expands possible precursors to a medley of phenols which often offer unique patterns of substitution in which halide analogs are not commonly available [12]. In parallel, 
the alkylating ability of sulfonates has led to their use in genetics to induce mutagenesis [14]. These mutagenic properties of sulfonates have recently prompted thorough investigation into sulfonate ester formation and hydrolysis by most major pharmaceutical companies [14].

As part of our ongoing interest in developing new methodologies for the synthesis of carbon-carbon bonds, we decided to synthesize a variety of sulfonate derivatives, which could be used for carbon-carbon bond forming reactions such as Sonogashira crosscoupling [12], Suzuki cross-coupling [15], Negishi cross-coupling [16], or carbon-oxygen bond forming reactions such as Ullman coupling [17]. We also wanted to make sure we chose sulfonyl chlorides with different substituents in order to confirm the versatility of sulfonates and their tolerance to other functional groups. As electrophilic partners, arylsulfonates have many advantages such as being readily available from phenol derivatives, ease of synthesis and purification, and their stability against hydrolysis. Herein we report a facile synthesis of arylsulfonates from sulfonyl chlorides and phenol derivatives. Using 2,4-dinitrophenyl 4-methylbenzenesulfonate and various amines we also report good regioselectivity for the $S_{N} A r$ products based on initial experiments.

\section{MATERIALS AND METHODS}

All reactions were carried out in oven-dried glassware under a nitrogen atmosphere. All the reagents were obtained from commercial sources and were used without further purification. Thinlayer chromatography was performed on silica gel plates containing fluorescent indicator. The crude arylsulfonate products were recrystallized in either $\mathrm{CH}_{2} \mathrm{Cl}_{2} /$ hexanes or hexane/ethyl acetate/ $\mathrm{H}_{2} \mathrm{O}$. ${ }^{1} \mathrm{H}-\mathrm{NMR}$ spectra (300 MHz) and ${ }^{13} \mathrm{C}-\mathrm{NMR}$ spectra (75 MHz) were recorded on a JEOL ECX300 spectrometer in $\mathrm{CDCl}_{3}$ or DMSO- $\mathrm{d}_{6}$. Chemical shifts are reported in parts per million (ppm, $\delta$ ) relative to the residual solvent peak, and coupling constants $(J)$ are reported in Hertz (Hz).

\section{Gas chromatography/mass spectrometry (GC/MS) conditions}

A Finnigan Focus Gas chromatography/Dual stage quadrupole (GC/DSQ) system with automated liquid sampler and Supelco Equity $515 \mathrm{~m} \mathrm{x} 0.25 \mathrm{~mm}$ x $0.25 \mu \mathrm{m}$ was used with a flow of ultrahigh purity He.
The temperatures for the aryl sulfonates in dichloromethane were inlet $220^{\circ} \mathrm{C}$, oven $50{ }^{\circ} \mathrm{C}$ for 2 minutes, and ramp to $270{ }^{\circ} \mathrm{C}$ at $15^{\circ} \mathrm{C} / \mathrm{min}$. The flow was set to $50 \mathrm{~mL} / \mathrm{min}$, the split ratio was $42: 1$, and the solvent delay was $1.05 \mathrm{~min}$. The mass scan range was $50-700 \mathrm{~m} / \mathrm{z}$. The syringe wash solvent was dichloromethane.

\section{General procedure for the preparation of arylsulfonates}

Phenol (0.941 g, $10 \mathrm{mmol}$ ) was dissolved in $10 \mathrm{~mL}$ of chilled dichloromethane. This was followed by the addition of pyridine $(1.6 \mathrm{~mL}, 20 \mathrm{mmol})$. The resulting solution was cooled in an ice bath under $\mathrm{N}_{2}$ atmosphere, followed by the addition of the sulfonyl chloride $(1.91 \mathrm{~g}, 10 \mathrm{mmol})$ portion-wise or dropwise. The mixture was stirred at $0{ }^{\circ} \mathrm{C}$ for 30 mins and then at room temperature (rt) for $12 \mathrm{~h}$. Reaction completion was verified by using thin layer chromatography (TLC) analysis. After dilution with $15 \mathrm{~mL}$ of $\mathrm{CH}_{2} \mathrm{Cl}_{2}$, the organic phase was washed with $\mathrm{H}_{2} \mathrm{O}$, brine and dried over anhydrous $\mathrm{Na}_{2} \mathrm{SO}_{4}$. After solvent evaporation, the residue was recrystallized from $\mathrm{CH}_{2} \mathrm{Cl}_{2} /$ hexanes or hexane/ethyl acetate $/ \mathrm{H}_{2} \mathrm{O}$ to afford the pure product in good to excellent yields.

\section{General procedure for the $\mathrm{S}_{\mathrm{N}} \mathrm{Ar}$ reaction}

2,4-Dinitrophenyl 4-methylbenzenesulfonate ( $0.423 \mathrm{~g}$, $1.25 \mathrm{mmol}$ ) and the amine (5 molar equiv) were dissolved in 1,2-dimethyoxyethane (DME) (0.1 M). This was followed by the addition of $4 \AA$ molecular sieves (320 $\mathrm{mg} / \mathrm{mmol})$. The mixture was flushed with $\mathrm{N}_{2}$ and stirred at $50{ }^{\circ} \mathrm{C}$ for $24 \mathrm{~h}$. The mixture was diluted with EtOAc followed by aqueous extraction. The organic layer was dried over $\mathrm{Na}_{2} \mathrm{SO}_{4}$ and evaporated. The crude product was purified by silica gel column chromatography in $\mathrm{CH}_{2} \mathrm{Cl}_{2}$.

Phenyl 4-methylbenzenesulfonate (3a): White powdery crystals $(93 \%), \mathrm{mp} 94-95{ }^{\circ} \mathrm{C} . R_{f}=0.28$ (n-hexane/ethyl acetate 4:1, v/v). ${ }^{1} \mathrm{H}$ NMR (300 $\mathrm{MHz}, \mathrm{CDCl}_{3}$ ): $\delta 7.67$ (d, 2H, Ar-H, $J=8.22 \mathrm{~Hz}$ ), 7.29-7.23 (m, 5H,), 6.95 (d, 2H, Ar-H, $J=8.25 \mathrm{~Hz}$ ), 2.42 (s, 3H). ${ }^{13} \mathrm{C}$ NMR (75 MHz, $\mathrm{CDCl}_{3}$ ): $\delta 149.75$, 145.39, 132.53, 129.82, 128.65, 128.56, 127.15, 122.46 and 21.78. HRMS (ESI): cald. For $\mathrm{C}_{13} \mathrm{H}_{12} \mathrm{NaO}_{3} \mathrm{~S}[\mathrm{M}+\mathrm{Na}]^{+}$271.0400; found 271.0399.

Phenyl 2-nitrobenzenesulfonate (3b): Yellow solids (85\%), mp 57-59 ${ }^{\circ} \mathrm{C} . R_{f}=0.17$ (n-hexane/ethyl acetate 4:1, v/v). ${ }^{1} \mathrm{H}$ NMR (300 MHz, $\mathrm{CDCl}_{3}$ ): $\delta 7.92$ 
(dd, 1H, Ar-H, $J=7.71,3.57 \mathrm{~Hz}$ ), 7.81 (dd, 1H, Ar-H, $J=6.87,3.84 \mathrm{~Hz}$ ), 7.80 (t, 1H, Ar-H, $J=7.95$ $\mathrm{Hz}$ ), 7.66 (ddd, $1 \mathrm{H}, \mathrm{Ar}-\mathrm{H}, J=7.68,7.68,2.73 \mathrm{~Hz}$ ), 7.35-7.27 (m, 2H, Ar-H), 7.17-7.16 (m, 3H, Ar-H). ${ }^{13} \mathrm{C}$ NMR (75 MHz, $\left.\mathrm{CDCl}_{3}\right): \delta 149.23,148.82,135.51$, 132.23, 132.06, 130.03, 128.46, 127.81, 124.93 and 122.31. HRMS (ESI): cald. For $\mathrm{C}_{12} \mathrm{H}_{9} \mathrm{NNaO}_{5} \mathrm{~S}$ $[\mathrm{M}+\mathrm{Na}]^{+}$302.0100; found 302.0094.

Phenyl 3-nitrobenzenesulfonate (3c): White powder (81\%), mp 93-96 ${ }^{\circ} \mathrm{C} . R_{f}=0.31$ (n-hexane/ethyl acetate 4:1, v/v). ${ }^{1} \mathrm{H}$ NMR (300 MHz, $\left.\mathrm{CDCl}_{3}\right): \delta 8.68(\mathrm{~d}, 1 \mathrm{H}$, Ar-H, $J=1.92 \mathrm{~Hz}$ ), 8.52 (dd, $1 \mathrm{H}$, Ar-H, $J=8.25$, $1.11 \mathrm{~Hz}$ ), 8.15 (dd, $1 \mathrm{H}$, Ar-H, $J=7.71,1.08 \mathrm{~Hz}$ ), 7.76 (ddd, $1 \mathrm{H}$, Ar-H, $J=8.16,7.98,1.38 \mathrm{~Hz}$ ), 7.35-7.28 (m, 3H, Ar-H), 7.02-6.99 (m, 2H, Ar-H). ${ }^{13} \mathrm{C}$ NMR (75 MHz, $\left.\mathrm{CDCl}_{3}\right): \delta$ 149.27, 148.32, 137.62, 133.93, 130.70, 130.10, 128.70, 127.84, 123.82 and 122.17. HRMS (ESI): cald. For $\mathrm{C}_{12} \mathrm{H}_{9} \mathrm{NNaO}_{5} \mathrm{~S}[\mathrm{M}+\mathrm{Na}]^{+}$302.0100; found 302.0094 .

Phenyl 4-nitrobenzenesulfonate (3d): Light pink crystals (88\%), mp $116-120{ }^{\circ} \mathrm{C} . R_{f}=0.62$ (nhexane/ethyl acetate 4:1, v/v). ${ }^{1} \mathrm{H}$ NMR (300 MHz, $\mathrm{CDCl}_{3}$ ): $\delta 8.35$ (d, 2H, Ar-H, $J=8.79 \mathrm{~Hz}$ ), 8.02 (d, 2H, Ar-H, $J=7.14 \mathrm{~Hz}$ ), 7.35-7.28 (m, 3H, Ar$\mathrm{H}), 7.00-6.97$ (m, 2H, Ar-H). ${ }^{13} \mathrm{C}$ NMR (75 MHz, $\left.\mathrm{CDCl}_{3}\right): \delta 151.08,149.31,141.09,130.09,129.99$, 127.83, 124.41 and 122.16. HRMS (ESI): cald. For $\mathrm{C}_{12} \mathrm{H}_{9} \mathrm{NNaO}_{5} \mathrm{~S}[\mathrm{M}+\mathrm{Na}]^{+}$302.0100; found 302.0094 .

Phenyl 2,4,5-trichlorobenzenesulfonate (3e): White crystals (56\%), mp 107-108 ${ }^{\circ} \mathrm{C} . R_{f}=0.60$ (nhexane/ethyl acetate 4:1, v/v). ${ }^{1} \mathrm{H}$ NMR (300 MHz, $\left.\mathrm{CDCl}_{3}\right): \delta 8.00$ (s, $\left.1 \mathrm{H}, \mathrm{Ar}-\mathrm{H}\right), 7.73(\mathrm{~s}, 1 \mathrm{H}, \mathrm{Ar}-\mathrm{H})$, 7.37-7.28 (m, 3H, Ar-H), 7.14-7.12 (m, 2H, Ar-H). ${ }^{13} \mathrm{C}$ NMR (75 MHz, $\mathrm{CDCl}_{3}$ ): $\delta$ 149.23, 139.69, 133.45, 133.40, 133.25, 132.14, 131.98, 130.13, 127.82 and 121.96. HRMS (ESI): cald. For $\mathrm{C}_{12} \mathrm{H}_{7} \mathrm{Cl}_{3} \mathrm{NaO}_{3} \mathrm{~S}[\mathrm{M}+\mathrm{Na}]^{+}$358.9100; found 358.9074 .

Phenyl methanesulfonate (3f): White powdery crystals $(97 \%)$, mp $62-64{ }^{\circ} \mathrm{C} . R_{f}=0.34$ (n-hexane/ ethyl acetate $4: 1, \mathrm{v} / \mathrm{v}) .{ }^{1} \mathrm{H}$ NMR (300 $\left.\mathrm{MHz}, \mathrm{CDCl}_{3}\right)$ : 反 7.45-7.42 (m, 2H, Ar-H), 7.35-7.27 (m, 3H, Ar-H), 3.14 (s, 3H). ${ }^{13} \mathrm{C}$ NMR (75 MHz, $\left.\mathrm{CDCl}_{3}\right): \delta 149.37$, 130.14, 127.52, 122.10 and 137.43. HRMS (ESI): cald. For $\mathrm{C}_{7} \mathrm{H}_{8} \mathrm{NaO}_{3} \mathrm{~S}[\mathrm{M}+\mathrm{Na}]^{+} 195.0100$; found 195.0086.
Phenyl 2,4,6-trimethylbenzenesulfonate (3g): White solid (82\%), mp 100-101 ${ }^{\circ} \mathrm{C} . R_{f}=0.69$ (nhexane/ethyl acetate 4:1, v/v). ${ }^{1} \mathrm{H}$ NMR $(300 \mathrm{MHz}$, DMSO- $\left.\mathrm{d}_{6}\right): \delta$ 7.37-7.34 (m, 3H, Ar-H) , 7.13 (s, 2H, Ar-H), 6.97 (d, 2H, Ar-H, J = $8.2 \mathrm{~Hz}$ ), 2.45 (s, 6H), 2.29 (s, 3H). ${ }^{13} \mathrm{C}$ NMR: $\delta\left(75 \mathrm{MHz}\right.$, DMSO- $\mathrm{d}_{6}$ ) 149.41, 144.80, 140.31, 132.39, 130.57, 130.15, 127.96, 122.39, 22.66 and 21.15. HRMS (ESI): cald. For $\mathrm{C}_{15} \mathrm{H}_{16} \mathrm{NaO}_{3} \mathrm{~S}[\mathrm{M}+\mathrm{Na}]^{+} 299.0700$; found 299.0681 .

Phenyl 2,4-dinitrobenzenesulfonate (3h): Yellow solid (74\%), mp $112-113{ }^{\circ} \mathrm{C} . R_{f}=0.31$ (n-hexane/ ethyl acetate 4:1, v/v). ${ }^{1} \mathrm{H}$ NMR (300 MHz, DMSO$\mathrm{d}_{6}$ ): $\delta 9.11$ (s, $\left.1 \mathrm{H}, \mathrm{Ar}-\mathrm{H}\right), 8.59$ (d, $1 \mathrm{H}, \mathrm{Ar}-\mathrm{H}, J=6.33$ $\mathrm{Hz}$ ), 8.23 (d, $1 \mathrm{H}, \mathrm{Ar}-\mathrm{H}, J=8.79 \mathrm{~Hz}), 7.45$ (m, 3H, Ar-H), 7.19 (m, 2H, Ar-H). ${ }^{13} \mathrm{C}$ NMR (75 MHz, DMSO- $\mathrm{d}_{6}$ ): $\delta 152.07,149.14,148.68,134.10,131.39$, 131.14, 128.96, 128.02, 122.47 and 121.64. HRMS (ESI): cald. For $\mathrm{C}_{12} \mathrm{H}_{8} \mathrm{~N}_{2} \mathrm{NaO}_{7} \mathrm{~S}[\mathrm{M}+\mathrm{Na}]^{+} 346.9900$; found 346.9891 .

3,5-Dimethylphenyl 4-methylbenzenesulfonate (3i): Yellowish-white fluffy crystals (90\%), mp 76-79 ${ }^{\circ} \mathrm{C} . R_{f}=0.71$ (n-hexane/ethyl acetate 4:1, v/v). ${ }^{1} \mathrm{H}$ NMR (300 MHz, $\mathrm{CDCl}_{3}$ ): $\delta 7.72(\mathrm{~d}, 2 \mathrm{H}$, Ar-H, $J=8.25 \mathrm{~Hz}$ ) , 7.30 (d, 2H, Ar-H, $J=8.22$ $\mathrm{Hz}$ ), 6.86 (s, 1H, Ar-H), 6.60 (s, 2H, Ar-H), 2.45 (s, 3H), 2.23 (s, 6H). ${ }^{13} \mathrm{C}$ NMR (75 MHz, $\mathrm{CDCl}_{3}$ ): $\delta$ 149.60, 145.22, 139.55, 132.83, 129.73, 128.79, 128.57, 119.88, 21.79 and 21.24. HRMS (ESI): cald. For $\mathrm{C}_{15} \mathrm{H}_{16} \mathrm{NaO}_{3} \mathrm{~S}[\mathrm{M}+\mathrm{Na}]^{+}$299.0700; found 299.0712 .

3,5-Dimethylphenyl 3-nitrobenzenesulfonate (3j): White fluffy crystals (64\%), mp 88-89 ${ }^{\circ} \mathrm{C} . R_{f}=0.33$ (n-hexane/ethyl acetate 4:1, v/v). ${ }^{1} \mathrm{H}$ NMR (300 $\mathrm{MHz}, \mathrm{CDCl}_{3}$ ): $\delta 8.70$ (t, $1 \mathrm{H}, \mathrm{Ar}-\mathrm{H}, J=1.80 \mathrm{~Hz}$ ), 8.52 (ddd, $1 \mathrm{H}$, Ar-H, $J=8.22,4.95,1.11 \mathrm{~Hz}$ ), 8.16 (ddd, $1 \mathrm{H}$, Ar-H, $J=7.98,4.95,1.11$ ), 7.77 (t, $1 \mathrm{H}, \mathrm{Ar}-\mathrm{H}, J=8.25 \mathrm{~Hz}$ ), 6.91-6.90 (m, 1H, Ar-H), 6.62 (s, 1H, Ar-H), 2.25 (s, 6H). ${ }^{13} \mathrm{C}$ NMR (75 $\left.\mathrm{MHz}, \mathrm{CDCl}_{3}\right): \delta 149.16,148.25,140.10,137.78$, 133.95, 130.70, 129.44, 128.63, 123.74, 119.54 and 21.23. HRMS (ESI): cald. For $\mathrm{C}_{14} \mathrm{H}_{13} \mathrm{NNaO}_{5} \mathrm{~S}$ $[\mathrm{M}+\mathrm{Na}]^{+} 330.0400$; found 330.0407.

3,5-Dimethylphenyl 4-nitrobenzenesulfonate (3k): Light yellow crystals (75\%), mp $115-118{ }^{\circ} \mathrm{C} . R_{f}=$ 0.68 (n-hexane/ethyl acetate 4:1, v/v). ${ }^{1} \mathrm{H}$ NMR (300 MHz, $\mathrm{CDCl}_{3}$ ): $\delta 8.37$ (d, 2H, Ar-H, $J=6.87 \mathrm{~Hz}$ ), 
8.06 (d, 2H, Ar-H, $J=6.87 \mathrm{~Hz}), 6.91$ (s, 1H, Ar-H), 6.63 (s, 2H, Ar-H), 2.25 (s, 6H). ${ }^{13} \mathrm{C}$ NMR (75 MHz, $\left.\mathrm{CDCl}_{3}\right): \delta 150.90,149.21,141.41,140.09,129.93$, 129.42, 124.34, 119.51 and 21.27. HRMS (ESI): cald. For $\mathrm{C}_{14} \mathrm{H}_{13} \mathrm{NNaO}_{5} \mathrm{~S}[\mathrm{M}+\mathrm{Na}]^{+} 330.0400$; found 330.0407 .

3,5-Dimethylphenyl 2,4,5-trichlorobenzenesulfonate (3l): Tan crystals (58\%), mp $110-112{ }^{\circ} \mathrm{C}, R_{f}=0.62$ (n-hexane/ethyl acetate 4:1, v/v). ${ }^{1} \mathrm{H}$ NMR (300 MHz, $\mathrm{CDCl}_{3}$ ): $\delta 8.04$ (s, 1H, Ar-H), 7.73 (s, 1H, Ar-H), 6.91 (s, 1H, Ar-H), 6.75 (s, 2H, Ar-H), 2.27 (s, 6H). ${ }^{13} \mathrm{C}$ NMR $\left(75 \mathrm{MHz}, \mathrm{CDCl}_{3}\right): \delta$ 149.09, 140.09, 139.51, 133.54, 133.38, 133.33, 132.06, 131.97, 129.46, 119.31 and 21.32. HRMS (ESI): cald. For $\mathrm{C}_{14} \mathrm{H}_{11} \mathrm{Cl}_{3} \mathrm{NaO}_{3} \mathrm{~S}$ [M $\left.+\mathrm{Na}\right]^{+}$386.9400; found 386.9371.

3,5-Dimethylphenyl methanesulfonate (3m): Yellow oil (64\%), $R_{f}=0.93$ (n-hexane/ethyl acetate 1:1, v/v). ${ }^{1} \mathrm{H}$ NMR $\left(300 \mathrm{MHz}, \mathrm{CDCl}_{3}\right): \delta 6.95$ (s, $1 \mathrm{H}, \mathrm{Ar}-\mathrm{H}), 6.89$ (s, 2H, Ar-H), 3.11 (s, 3H), 2.32 (s, 6H). ${ }^{13} \mathrm{C}$ NMR $\left(75 \mathrm{MHz}, \mathrm{CDCl}_{3}\right): \delta 149.34$, 140.10, 129.15, 119.51, 37.38 and 21.29. HRMS (ESI): cald. For $\mathrm{C}_{9} \mathrm{H}_{12} \mathrm{NaO}_{3} \mathrm{~S}[\mathrm{M}+\mathrm{Na}]^{+}$223.0400; found 223.0405.

3,5-Dimethylphenyl 2,4,6-trimethylbenzenesulfonate (3n): White fluffy crystals (76\%), mp $109-111^{\circ} \mathrm{C} . R_{f}=0.57$ (n-hexane/ethyl acetate 4:1, v/v). ${ }^{1} \mathrm{H}$ NMR (300 MHz, $\mathrm{CDCl}_{3}$ ): $\delta 6.97$ (s, $2 \mathrm{H}$, Ar-H) , 6.84 (s, 1H, Ar-H), 6.60 (s, 2H, Ar-H), 2.57 (s, 6H), 2.32 (s, 3H), 2.22 (s, 6H). ${ }^{13} \mathrm{C}$ NMR (75 $\mathrm{MHz}, \mathrm{CDCl}_{3}$ ): $\delta 149.41,143.74,140.45,139.51$, 131.76, 130.98, 128.64, 119.69, 22.87, 21.25 and 21.19. HRMS (ESI): cald. For $\mathrm{C}_{17} \mathrm{H}_{20} \mathrm{NaO}_{3} \mathrm{~S}[\mathrm{M}+$ $\mathrm{Na}]^{+}$327.1000; found 327.1025.

2-Chlorophenyl 4-methylbenzenesulfonate (30): White powdery crystals (89\%), mp 73-74 ${ }^{\circ} \mathrm{C} . R_{f}=$ 0.45 (n-hexane/ethyl acetate 4:1, v/v). ${ }^{1} \mathrm{H}$ NMR (300 MHz, $\mathrm{CDCl}_{3}$ ): $\delta 7.77$ (d, 2H, Ar-H, $J=8.22$ $\mathrm{Hz}$ ), 7.36-7.30 (m, 3H, Ar-H), 7.28-7.25 (m, $1 \mathrm{H}$, Ar-H), 7.23-7.21 (m, 1H, Ar-H), 7.19-7.17 (m, 1H, Ar-H), 2.45 (s, 3H). ${ }^{13} \mathrm{C}$ NMR (75 MHz, $\left.\mathrm{CDCl}_{3}\right): \delta 145.74,132.75,131.41,130.83,129.85$, 128.74, 127.96, 127.87, 127.71, 124.35 and 21.85. HRMS (ESI): cald. For $\mathrm{C}_{13} \mathrm{H}_{11} \mathrm{ClNaO}_{3} \mathrm{~S}[\mathrm{M}+$ $\mathrm{Na}]^{+}$305.0000; found 305.0010.

2-Nitrophenyl 4-methylbenzenesulfonate (3p): Tan powdery crystals (73\%), mp 79-82 ${ }^{\circ} \mathrm{C} . R_{f}=0.34$ (n-hexane/ethyl acetate $4: 1, \mathrm{v} / \mathrm{v}) .{ }^{1} \mathrm{H}$ NMR (300 MHz,
$\left.\mathrm{CDCl}_{3}\right): \delta 7.90$ (dd, $1 \mathrm{H}, \mathrm{Ar}-\mathrm{H}, J=7.90,1.65 \mathrm{~Hz}$ ), 7.77 (d, $2 \mathrm{H}, \mathrm{Ar}-\mathrm{H}, J=8.52 \mathrm{~Hz}), 7.65-7.59$ (m, $2 \mathrm{H}$, Ar-H), 7.47-7.39 (m, 1H, Ar-H), 7.34 (d, 2H, Ar-H, $J=8.52 \mathrm{~Hz}), 2.47$ (s, 3H, $\left.-\mathrm{CH}_{3} \mathrm{Ar}\right) .{ }^{13} \mathrm{C}$ NMR $(75$ $\left.\mathrm{MHz}, \mathrm{CDCl}_{3}\right): \delta 146.39,142.85,141.58,134.28$, 131.60, 130.12, 128.77, 127.62, 125.90, 125.47 and 21.90. HRMS (ESI): cald. For $\mathrm{C}_{13} \mathrm{H}_{11} \mathrm{NNaO}_{5} \mathrm{~S}$ [M $+\mathrm{Na}]^{+}$316.0300; found 316.0250.

3-Nitrophenyl 4-methylbenzenesulfonate (3q): White crystals (83\%), mp 109-110 ${ }^{\circ} \mathrm{C} . R_{f}=0.35$ (n-hexane/ethyl acetate 4:1, v/v). ${ }^{1} \mathrm{H}$ NMR (300 $\mathrm{MHz}, \mathrm{CDCl}_{3}$ ): $\delta 8.12$ (ddd, $1 \mathrm{H}, \mathrm{Ar}-\mathrm{H}, J=8.22$, 2.19, $1.11 \mathrm{~Hz}$ ), 7.79 (t, $1 \mathrm{H}, \mathrm{Ar}-\mathrm{H}, J=2.19 \mathrm{~Hz}$ ), 7.73 (d, 2H, Ar-H, $J=8.52 \mathrm{~Hz}$ ), 7.52 (t, $1 \mathrm{H}, \mathrm{Ar}-$ $\mathrm{H}, J=8.22 \mathrm{~Hz}$ ), 7.42 (ddd, $1 \mathrm{H}, \mathrm{Ar}-\mathrm{H}, J=8.22$, 2.46, $1.11 \mathrm{~Hz}$ ), 7.35 (d, 2H, Ar-H, $J=8.49 \mathrm{~Hz}$ ), 2.47 (s, 3H, $-\mathrm{CH}_{3} \mathrm{Ar}$ ). ${ }^{13} \mathrm{C}$ NMR (75 MHz, $\mathrm{CDCl}_{3}$ ): $\delta$ $149.81,148.78,146.36,131.72,130.49,130.22$, 128.93, 128.60, 122.09, 118.06 and 21.87. HRMS (ESI): cald. For $\mathrm{C}_{13} \mathrm{H}_{11} \mathrm{NNaO}_{5} \mathrm{~S}[\mathrm{M}+\mathrm{Na}]^{+} 316.0300$; found 316.0250.

4-Nitrophenyl 4-methylbenzenesulfonate (3r): White-yellowish crystals (82\%), mp 97-98 ${ }^{\circ} \mathrm{C}$. $R_{f}=0.36$ (n-hexane/ethyl acetate 4:1, v/v). ${ }^{1} \mathrm{H}$ NMR (300 MHz, $\mathrm{CDCl}_{3}$ ): $\delta 8.18$ (d, 2H, Ar-H, $J=9.06$ $\mathrm{Hz}$ ), 7.72 (d, 2H, Ar-H, $J=8.52 \mathrm{~Hz}$ ), 7.35 (d, 2H, Ar-H, $J=8.52 \mathrm{~Hz}$ ), 7.18 (d, 2H, Ar-H, $J=9.09$ $\mathrm{Hz}), 2.46$ (s, 3H). ${ }^{13} \mathrm{C}$ NMR (75 $\mathrm{MHz}, \mathrm{CDCl}_{3}$ ): $\delta$ 154.00, 146.37, 146.29, 131.78, 130.21, 128.56, 125.49, 123.32 and 21.86. HRMS (ESI): cald. For $\mathrm{C}_{13} \mathrm{H}_{11} \mathrm{NNaO}_{5} \mathrm{~S}[\mathrm{M}+\mathrm{Na}]^{+}$316.0300; found 316.0250 .

2-Chlorophenyl 2,4,6-trimethylbenzenesulfonate (3s): White crystals (81\%), mp 66-69 ${ }^{\circ} \mathrm{C} . R_{f}=0.94$ (n-hexane/ethyl acetate 1:1, v/v). ${ }^{1} \mathrm{H}$ NMR $(300 \mathrm{MHz}$, $\left.\mathrm{CDCl}_{3}\right): \delta$ 7.41-7.35 (m, $\left.1 \mathrm{H}, \mathrm{Ar}-\mathrm{H}\right), 7.20-7.16(\mathrm{~m}$, 2H, Ar-H), 7.10-7.06 (m, 1H, Ar-H), 6.98 (s, 2H, Ar-H), 2.58 (s, 6H), 2.33 (s, 3H). ${ }^{13} \mathrm{C}$ NMR (75 MHz, $\left.\mathrm{CDCl}_{3}\right): \delta 145.77,144.21,140.76,140.63,131.96$, 131.41, 130.96, 127.93, 127.80, 124.04, 23.03 and 21.25. HRMS (ESI): cald. For $\mathrm{C}_{15} \mathrm{H}_{15} \mathrm{ClNaO}_{3} \mathrm{~S}$ [M $+\mathrm{Na}^{+}$333.0300; found 333.0310.

2-Nitrophenyl 2,4,6-trimethylbenzenesulfonate (3t): Yellow powdery crystals (70\%), mp $100-103^{\circ} \mathrm{C}$. $R_{f}=0.37$ (n-hexane/ethyl acetate 4:1, v/v). ${ }^{1} \mathrm{H}$ NMR $\left(300 \mathrm{MHz}, \mathrm{CDCl}_{3}\right): \delta 7.89$ (dd, $1 \mathrm{H}, \mathrm{Ar}-\mathrm{H}, J=1.65$ $\mathrm{Hz}, 7.98 \mathrm{~Hz}$ ), 7.57 (td, $1 \mathrm{H}, \mathrm{Ar}-\mathrm{H}, J=8.52 \mathrm{~Hz}$, 8.25), 7.40 (td, $1 \mathrm{H}, \mathrm{Ar}-\mathrm{H}, J=8.25 \mathrm{~Hz}, 7.68 \mathrm{~Hz}$ ), 
7.29 (dd, 1H, Ar-H, $J=1.35$ Hz), 7.00 (s, 2H, Ar$\mathrm{H}), 2.55$ (s, 6H), $2.34(3 \mathrm{H}) .{ }^{13} \mathrm{C}$ NMR $(75 \mathrm{MHz}$, $\mathrm{CDCl}_{3}$ ): $\delta 144.79,143.51,141.54,140.77,133.99$, 132.12, 130.18, 127.42, 125.78, 125.41, 22.88 and 21.27. HRMS (ESI): cald. For $\mathrm{C}_{15} \mathrm{H}_{15} \mathrm{NNaO}_{5} \mathrm{~S}$ [M $+\mathrm{Na}]^{+}$344.0600; found 344.0563.

3-Nitrophenyl 2,4,6-trimethylbenzenesulfonate (3u): White crystals (74\%), mp 138-139 ${ }^{\circ} \mathrm{C}$, $R_{f}=0.38$ (n-hexane/ethyl acetate 4:1, v/v). ${ }^{1} \mathrm{H}$ NMR (300 MHz, $\mathrm{CDCl}_{3}$ ): $\delta 8.12$ (1H, ddd, Ar-H, $J=9.06$, 1.11, $1.08 \mathrm{~Hz}$ ), 7.77 (t, $1 \mathrm{H}$, Ar-H, $J=2.19 \mathrm{~Hz}$ ), 7.51 (t, $1 \mathrm{H}, \mathrm{Ar}-\mathrm{H}, J=8.25 \mathrm{~Hz}), 7.43-7.39(\mathrm{~m}, 1 \mathrm{H}$, Ar-H), 7.01 (s, 2H, Ar-H), 2.57 (s, 6H), 2.34 (s, 3H). ${ }^{13} \mathrm{C}$ NMR $\left(75 \mathrm{MHz}, \mathrm{CDCl}_{3}\right): \delta 149.79$, 148.82, 144.83, 140.64, 132.19, 130.45, 129.89, 128.85, 121.96, 117.88, 22.87 and 21.29. HRMS (ESI): cald. For $\mathrm{C}_{15} \mathrm{H}_{15} \mathrm{NNaO}_{5} \mathrm{~S}[\mathrm{M}+\mathrm{Na}]^{+}$ 344.0600; found 344.0563.

4-Nitrophenyl 2,4,6-trimethylbenzenesulfonate (3v): Light brown crystals (77\%), mp $115-116^{\circ} \mathrm{C}$, $R_{f}=0.42$ (n-hexane/ethyl acetate $\left.4: 1, \mathrm{v} / \mathrm{v}\right) .{ }^{1} \mathrm{H}$ NMR (300 MHz, $\left.\mathrm{CDCl}_{3}\right): \delta 8.18(\mathrm{~d}, 2 \mathrm{H}, \mathrm{Ar}-\mathrm{H}$, $J=9.36 \mathrm{~Hz}), 7.15(\mathrm{~d}, 2 \mathrm{H}, \mathrm{Ar}-\mathrm{H}, J=9.09 \mathrm{~Hz})$, 7.00 (s, 2H, Ar-H), 2.57 (s, 6H), 2.34 (s, 3H). ${ }^{13} \mathrm{C}$ NMR (75 MHz, $\mathrm{CDCl}_{3}$ ): $\delta 154.00,146.16,144.75$, 140.58, 132.14, 130.03, 125.45, 123.13, 22.85 and 21.25. HRMS (ESI): cald. For $\mathrm{C}_{15} \mathrm{H}_{15} \mathrm{NNaO}_{5} \mathrm{~S}$ [M $+\mathrm{Na}^{+}$344.0600; found 344.0563.

2,4-Dinitrophenyl 2,4,6-trimethylbenzenesulfonate (3w): Milky yellow crystals (51\%), mp 121-123 ${ }^{\circ} \mathrm{C}$. $R_{f}=0.80$ (n-hexane/ethyl acetate $\left.1: 1, \mathrm{v} / \mathrm{v}\right) .{ }^{1} \mathrm{H}$ NMR (300 MHz, CDCl $)_{3}$ ): $\delta 8.76$ (d, $1 \mathrm{H}, \mathrm{Ar}-\mathrm{H}$, $J=2.76 \mathrm{~Hz}$ ), 8.44 (dd, 1H, Ar-H, $J=8.79,2.76$ $\mathrm{Hz}$ ), 7.57 (d, 1H, Ar-H, $J=8.91$ ), 7.04 (s, 2H, Ar$\mathrm{H}), 2.57$ (s, 6H), 2.36 (s, 3H). ${ }^{13} \mathrm{C}$ NMR (75 MHz, $\left.\mathrm{CDCl}_{3}\right): \delta 146.19,145.64,145.16,142.95,140.89$, 132.38, 129.67, 128.46, 126.22, 121.61, 22.91 and 21.35. HRMS (ESI): cald. For $\mathrm{C}_{15} \mathrm{H}_{14} \mathrm{~N}_{2} \mathrm{NaO}_{7} \mathrm{~S}$ $[\mathrm{M}+\mathrm{Na}]^{+}$389.0400; found 389.0410.

N-Allyl-2,4-dinitroaniline (6a): Light yellowish powder (84\%). $R_{f}=0.61\left(\mathrm{CH}_{2} \mathrm{Cl}_{2}\right) .{ }^{1} \mathrm{H}$ NMR (300 $\mathrm{MHz}, \mathrm{CDCl}_{3}$ ): $\delta 9.15$ (d, $1 \mathrm{H}, \mathrm{Ar}-\mathrm{H}, J=2.76 \mathrm{~Hz}$ ), 8.69 (br s, $1 \mathrm{H}, \mathrm{NH}$ ), 8.27 (dd, $1 \mathrm{H}, \mathrm{Ar}-\mathrm{H}, J=9.63$, $2.76 \mathrm{~Hz}), 6.91$ (d, $1 \mathrm{H}, \mathrm{Ar}-\mathrm{H}, J=9.63 \mathrm{~Hz}), 5.95$ (ddt, $1 \mathrm{H},=\mathrm{CH}, J=17.31,10.18,6.19 \mathrm{~Hz}$ ), 5.36 (dd, $1 \mathrm{H},=\mathrm{CH}_{\text {trans }}, J=17.31,1.93 \mathrm{~Hz}$ ), 5.31 (dd, $\left.1 \mathrm{H},=\mathrm{CH}_{\text {cis }}, J=10.18,1.93 \mathrm{~Hz}\right), 4.11(\mathrm{dd}, 1 \mathrm{H}$,
$\mathrm{NCH}_{2}, J=5.50,1.65 \mathrm{~Hz}$ ), 4.07 (dd, $1 \mathrm{H}, \mathrm{NCH}_{2}$, $J=5.50,1.65 \mathrm{~Hz}) .{ }^{13} \mathrm{C} \mathrm{NMR}\left(75 \mathrm{MHz}, \mathrm{CDCl}_{3}\right): \delta$ 151.6, 137.2, 135.4, 135.3, 130.7, 120.8, 117.4, 115.2 and 45.1. HRMS (ESI): cald. For $\mathrm{C}_{9} \mathrm{H}_{9} \mathrm{~N}_{3} \mathrm{NaO}_{4}$ $[\mathrm{M}+\mathrm{Na}]^{+}$246.0500; found 246.0490.

N-Butyl-2,4-dinitroaniline (6b): Light yellow powder (81\%). $R_{f}=0.65\left(\mathrm{CH}_{2} \mathrm{Cl}_{2}\right) \cdot{ }^{1} \mathrm{H}$ NMR $(300$ $\left.\mathrm{MHz}, \mathrm{CDCl}_{3}\right): \delta 9.14(\mathrm{~d}, 1 \mathrm{H}, \mathrm{Ar}-\mathrm{H}, J=2.48 \mathrm{~Hz}$ ), 8.56 (br s, $1 \mathrm{H}, \mathrm{NH}$ ), 8.27 (dd, $1 \mathrm{H}, \mathrm{Ar}-\mathrm{H}, J=9.63$, $2.48 \mathrm{~Hz}), 6.92$ (d, $1 \mathrm{H}, \mathrm{Ar}-\mathrm{H}, J=9.63 \mathrm{~Hz}$ ), 3.43 (dt, $1 \mathrm{H}, \mathrm{NCH}_{2}, J=7.17,5.23 \mathrm{~Hz}$ ), 3.40 (dt, $1 \mathrm{H}$, $\left.\mathrm{NCH}_{2}, J=7.15,5.23 \mathrm{~Hz}\right), 1.82-1.72(\mathrm{~m}, 2 \mathrm{H}$, $\left.\mathrm{CH}_{2}\right), 1.56-1.44\left(\mathrm{~m}, 2 \mathrm{H}, \mathrm{CH}_{2}\right), 1.01\left(\mathrm{t}, 3 \mathrm{H}, \mathrm{CH}_{3}\right.$, $J=7.43 \mathrm{~Hz}) .{ }^{13} \mathrm{C}$ NMR $\left(75 \mathrm{MHz}, \mathrm{CDCl}_{3}\right): \delta 151.4$, 137.3, 135.2, 130.8, 120.8, 115.4, 50.1, 26.0, 20.2 and 13.8. HRMS (ESI): cald. For $\mathrm{C}_{10} \mathrm{H}_{13} \mathrm{~N}_{3} \mathrm{NaO}_{4}$ $[\mathrm{M}+\mathrm{Na}]^{+}$262.0800; found 262.0810.

N-Allyl-4-methylbenzenesulfonamide (7a): Brown powder (11\%), $R_{f}=0.32\left(\mathrm{CH}_{2} \mathrm{Cl}_{2}\right) .{ }^{1} \mathrm{H}$ NMR (300 $\left.\mathrm{MHz}, \mathrm{CDCl}_{3}\right): \delta 7.75$ (d, 2H, Ar-H, $J=8.26 \mathrm{~Hz}$ ), 7.31 (d, 2H, Ar-H, $J=8.26 \mathrm{~Hz}$ ), 5.70 (ddt, $1 \mathrm{H}$, $=\mathrm{CH}, J=17.34,10.18,6.05 \mathrm{~Hz}), 5.16(\mathrm{dd}, 1 \mathrm{H}$, $\left.=\mathrm{CH}_{\text {trans }}, J=17.34,1.93 \mathrm{~Hz}\right), 5.09(\mathrm{dd}, 1 \mathrm{H}$, $\left.=\mathrm{CH}_{\text {cis }}, J=10.18,1.93 \mathrm{~Hz}\right), 4.54($ app t, $1 \mathrm{H}, \mathrm{NH}$, $J=5.63 \mathrm{~Hz}$ ), 3.58 (app t, 2H, $\mathrm{NCH}_{2}, J=6.05 \mathrm{~Hz}$ ), $2.43(\mathrm{~s}, 3 \mathrm{H}) \cdot{ }^{13} \mathrm{C}$ NMR (75 MHz, $\mathrm{CDCl}_{3}$ ): $\delta$ 137.6, 136.2, 134.3, 129.4, 128.3, 117.4, 43.8 and 21.3. HRMS (ESI): cald. For $\mathrm{C}_{10} \mathrm{H}_{13} \mathrm{NNaO}_{2} \mathrm{~S}$ [M $+\mathrm{Na}]^{+}$234.0600; found 234.0610.

N-Allyl-4-methylbenzenesulfonamide (7b): Brown powder (8\%), $R_{f}=0.34\left(\mathrm{CH}_{2} \mathrm{Cl}_{2}\right) .{ }^{1} \mathrm{H}$ NMR (300 $\left.\mathrm{MHz}, \mathrm{CDCl}_{3}\right): \delta 7.74(\mathrm{~d}, 2 \mathrm{H}, \mathrm{Ar}-\mathrm{H}, J=8.26 \mathrm{~Hz})$, 7.30 (d, $2 \mathrm{H}, \mathrm{Ar}-\mathrm{H}, J=8.26 \mathrm{~Hz}$ ), 4.41 (app t, $1 \mathrm{H}$, $\mathrm{NH}, J=6.05 \mathrm{~Hz}$ ), 2.92 (app q, 2H, $\mathrm{NCH}_{2}, J=$ $6.87 \mathrm{~Hz}$ ), 2.42 (s, $\left.3 \mathrm{H}, \mathrm{Ar}-\mathrm{CH}_{3}\right), 1.48-1.38(\mathrm{~m}, 2 \mathrm{H}$, $\mathrm{CH}_{2}$ ), 1.34-1.22 (m, $\left.2 \mathrm{H}, \mathrm{CH}_{2}\right), 0.84$ (t, $3 \mathrm{H}, \mathrm{CH}_{3}$, $J=7.29 \mathrm{~Hz}) \cdot{ }^{13} \mathrm{C}$ NMR $\left(75 \mathrm{MHz}, \mathrm{CDCl}_{3}\right): \delta$ 137.6, 137.2, 129.3, 128.2, 42.5, 31.3, 21.4, 19.8 and 13.8. HRMS (ESI): cald. For $\mathrm{C}_{11} \mathrm{H}_{17} \mathrm{NNaO}_{2} \mathrm{~S}$ $[\mathrm{M}+\mathrm{Na}]^{+}$250.0900; found 250.0890.

\section{RESULTS AND DISCUSSION}

In our optimization experiments, the phenol was kept at 1.0 equivalent while the number of equivalents was varied for the sulfonyl chloride from 0.5 equivalents to 1.5 equivalents (Figure 1). The reaction was carried out using 2.0 equivalents of pyridine 


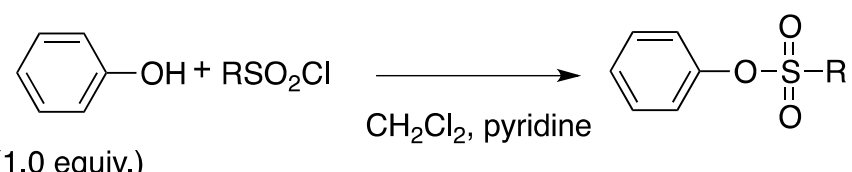

(1.0 equiv.)
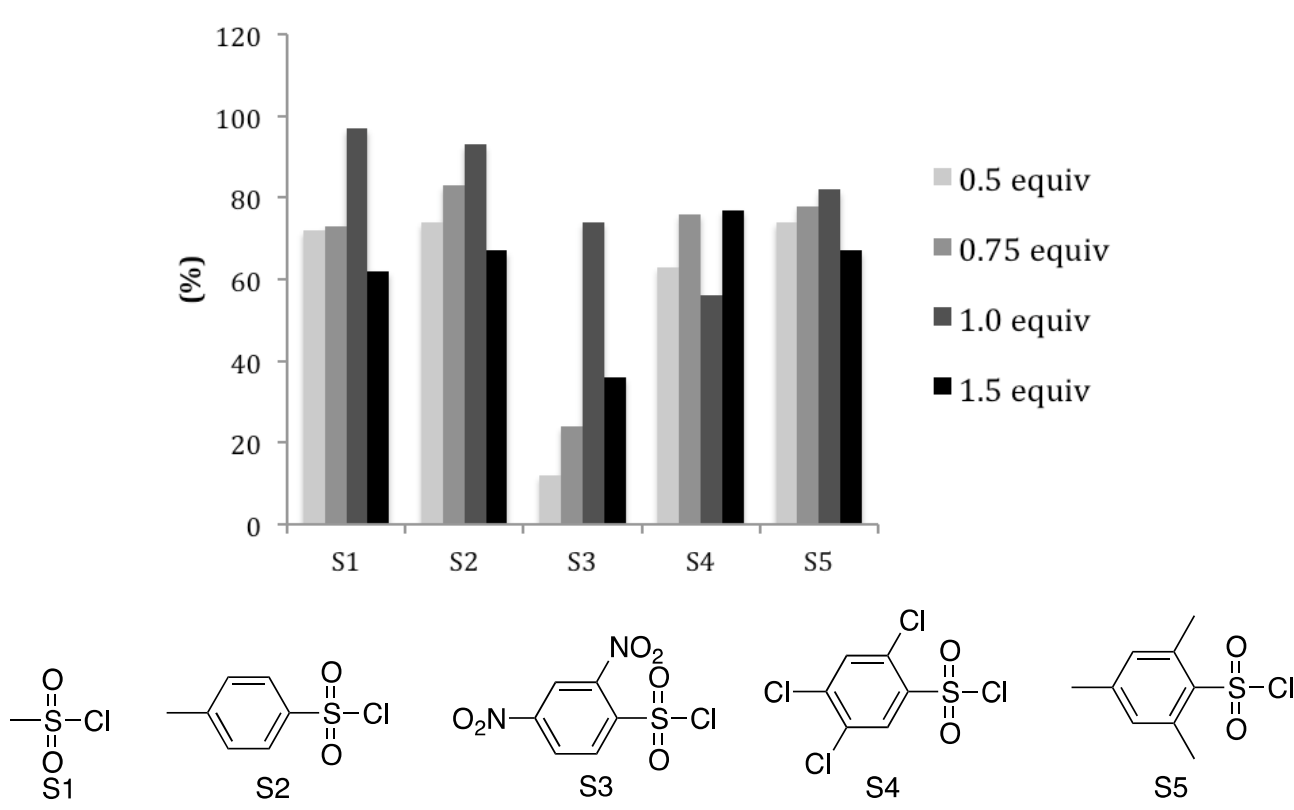

Figure 1. Varying the number of equivalents for each sulfonyl chloride.

in $\mathrm{CH}_{2} \mathrm{Cl}_{2}$ under $\mathrm{N}_{2}$ atmosphere at rt for $12 \mathrm{~h}$. Pyridine was used as a base because it does not result in hydrolysis of the sulfonate ester, which could occur with stronger hydroxide bases. The sulfonation synthesis also uses less expensive solvents as dichloromethane was used in all reactions.

For initial experiments, we selected methanesulfonyl chloride because it is the simplest sulfonyl chloride that was readily available (S1, Figure 1). As exemplified in the reaction of methanesulfonyl chloride with phenol (Figure 1), using 1.0 equivalent of methanesulfonyl chloride proved to be the most effective as it afforded the highest yield of the desired sulfonate product (97\%). The yields using 0.5 and 0.75 equivalents of methanesulfonyl chlorides were comparable $(72 \%$ and $73 \%$, respectively, Figure 1). When the number of equivalents of methanesulfonyl chloride was increased to 1.5 equivalents, the yield of the desired sulfonate product dropped to $62 \%$, with a substantial amount of the corresponding chloride recovered. Although we obtained the desired sulfonate product using various equivalents of methanesulfonyl chloride (0.5-1.5 equiv., Figure 1), all attempts to recrystallize the crude product using different solvent combinations were unsuccessful. Therefore, the reported yields were obtained after column chromatography purification of the crude product to obtain the pure product.

However, we were more interested in facile synthesis that would circumvent the use of column chromatopraphy as a purification technique. To that end, we chose another readily available and easyto-handle sulfonyl chloride, para-toluenesulfonyl chloride (S2, Figure 1), as the electrophilic partner for nucleophilic substitution with phenol. Here again, consistent with the results from the use of methanesulfonyl chloride, the use of 1.0 equivalent of para-toluenesulfonyl chloride proved to be the most effective as it resulted in the best yield (93\%) of the desired product. It is important to note that the crude product was purified by recrystallization to afford the pure product as a crystalline solid. Overall, the yields were good for each equivalent of para-toluenesulfonyl chloride that reacted with phenol. The yield of isolated product steadily increased from 0.5 equivalent (74\%), through 0.75 equivalent (83\%), to 1.0 equivalent (93\%). When the number 
of equivalents of para-toluenesulfonyl chloride was increased to 1.5 equivalent, the yield of the desired product was reduced to $67 \%$. Next, 2,4dinitrobenzene-1-sulfonyl chloride (S3, Figure 1) was reacted with phenol. The isolated yields of the desired product after recrystallization were as follows: $12 \%$ ( 0.5 equiv.), $24 \%$ ( 0.75 equiv.), $74 \%$ (1.0 equiv.), and 36\% (1.5 equiv.). As can be seen in this case (S3, Figure 1), the yields are the lowest, albeit the results are consistent with the highest yield of isolated product obtained with 1.0 equivalent of para-toluenesulfonyl chloride. As the number of equivalents of para-toluenesulfonyl chloride increased, the yield of desired product steadily increased until 1.0 equivalent and then dropped when the number of equivalents was increased to 1.5 equivalents. The two nitro groups at the ortho and para positions of 2,4-dinitrobenzene-1-sulfonyl chloride (S3, Figure 1) function as electron withdrawing groups via resonance. In the case of 2,4,5-trichlorobenzene-1-sulfonyl chloride (S4, Figure 1), there are three chlorine atoms at the ortho, meta, and para positions. These chlorine atoms function as electron withdrawing groups through inductive effect. The isolated yields of the desired product for 2,4,5-trichlorobenzene-1-sulfonyl chloride (S4, Figure 1) were 63\% (0.5 equiv.), $76 \%$ (0.75 equiv.), $56 \%$ (1.0 equiv.), and $77 \%$ (1.5 equiv.). As can be seen (S4, Figure 1), the results are different in that the highest yield of isolated product after recrystallization was obtained when 1.5 equivalents of 2,4,5-trichlorobenzene-1-sulfonyl chloride was used, and the lowest yield when 1.0 equivalents of 2,4,5-trichlorobenzene-1-sulfonyl chloride was used. The yield of isolated product was also comparable between 0.75 equivalents and 1.5 equivalents $(76 \%$ and $77 \%$, respectively, Figure 1). Finally, 2,4,6-trimethylbenzene-1-sulfonyl chloride (S5, Figure 1) was used as the electrophilic partner in nucleophilic substitution reaction with phenol. In 2,4,6-trimethylbenzene-1-sulfonyl chloride (S5, Figure 1), there are three methyl groups at the ortho and para positions functioning as electron donating groups through inductive effect. The percentages of product for 2,4,6-trimethylbenzene-1sulfonyl chloride (S5, Figure 1) were 74\% (0.5 equiv.), $78 \%$ (0.75 equiv.), $82 \%$ (1.0 equiv.), and $67 \%$ (1.5 equiv.). As shown in the use of 2,4,6trimethylbenzene-1-sulfonyl chloride (S5, Figure 1), the yield of the isolated product increased as the number of equivalents of 2,4,6-trimethylbenzene1-sulfonyl chloride increased and was consistent with S1, S2 and S3 in that the highest yield of the isolated product was obtained using 1.0 equivalents of 2,4,6-trimethylbenzene-1-sulfonyl chloride (S5, Figure 1). Overall, the use of 1.0 equivalent of the electrophilic sulfonyl chloride partner afforded the highest yield of isolated product in all cases except S4, where the highest yield was obtained with 1.5 equivalents of 2,4,5-trichlorobenzene-1sulfonyl chloride. In general, electron-rich, electrondeficient, and sterically hindered sulfonyl chlorides represent compatible electrophilic partners for nucleophilic substitution reaction with phenol. The use of 1.0 equivalent of phenol, 1.0 equivalent of sulfonyl chloride, 2.0 equivalents of pyridine in $\mathrm{CH}_{2} \mathrm{Cl}_{2}$ were chosen as the optimized reaction condition.

Under the optimized reaction conditions, various sulfonyl chloride derivatives (1) with both electrondonating and electron-withdrawing substituents were reacted with a diverse set of phenols (2) to establish the versatility of our sulfonation reaction (Table 1). Under the reaction conditions, generally good to excellent yields were obtained (Table 1 , entries 1-23).

The reaction tolerated a variety of sulfonyl chloride derivatives and phenols containing either an electrondonating group $\left(-\mathrm{CH}_{3}\right)$ or electron-withdrawing group $\left(-\mathrm{Cl}\right.$ and $-\mathrm{NO}_{2}$ ) on the phenyl ring.

Using the optimized reaction conditions, the substrate scope was investigated starting with a variation of the sulfonyl chloride partner and using phenol as the nucleophile (Table 1, entries 1-8). Sulfonyl chlorides 1a-1h bearing electron-donating or electron-withdrawing groups on the benzene ring reacted with phenol to give good to excellent yields (Table 1, entries 1-8). We believe electronic effect and steric effect of the substituents on the benzene ring may have an influence on the yield of the recovered products. The recovered crude products were purified by recrystallization to obtain the isolated yields reported in table 1 . It is not clear which factor, electronic effect or steric hindrance of the substituents on the benzene ring, is the main factor responsible for the difference in product yield. Aryl sulfonyl chlorides bearing electron-donating groups in the ortho and para positions reacted with phenol to give $93 \%$ and 
Table 1. Synthesis of sulfonates from sulfonyl chloride derivatives and phenol.

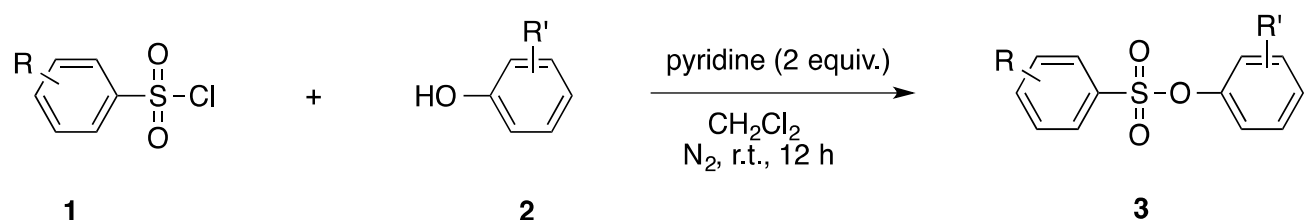

\begin{tabular}{|c|c|c|c|c|}
\hline Entry & Sulfonyl chloride $\mathbf{1}$ & Phenol 2 & Product 3 & Yield $^{\mathrm{a}} \% \%$ \\
\hline 1 & Ö & $2 a$ & & 93 \\
\hline 2 & & $2 \mathbf{a}$ & $\begin{array}{r}\mathrm{NO}_{2} \\
\mathbf{3 b}\end{array}$ & 85 \\
\hline 3 & $\mathrm{O}_{2} \mathrm{~N}_{1 \mathrm{c}}$ & $2 \mathbf{a}$ & & 81 \\
\hline 4 & -S & $2 \mathbf{a}$ & & 88 \\
\hline 5 & ${ }_{1 e} \mathrm{Cl}$ & $2 a$ & & 56 \\
\hline $6^{\mathrm{b}}$ & $\begin{array}{l}\mathrm{O} \\
-\stackrel{11}{S}-\mathrm{Cl} \\
0 \\
0 \\
\mathbf{1 f}\end{array}$ & $2 a$ & $\mathrm{O}_{3 \mathbf{f}}$ & 97 \\
\hline 7 & $\begin{array}{l}-S-C l \\
0 \\
0\end{array}$ & $2 \mathbf{a}$ & $3 \mathrm{~g}$ & 82 \\
\hline 8 & $\underbrace{\mathrm{S}}_{1 \mathrm{~h} \mathrm{NO}_{2}}-\mathrm{Cl}$ & $\mathbf{2 a}$ & $\begin{array}{r}\mathrm{NO}_{2} \\
3 \mathbf{h} \\
\end{array}$ & 74 \\
\hline 9 & $1 \mathrm{a}$ & $\mathrm{HO}$ & & 90 \\
\hline
\end{tabular}


Table 1 continued..

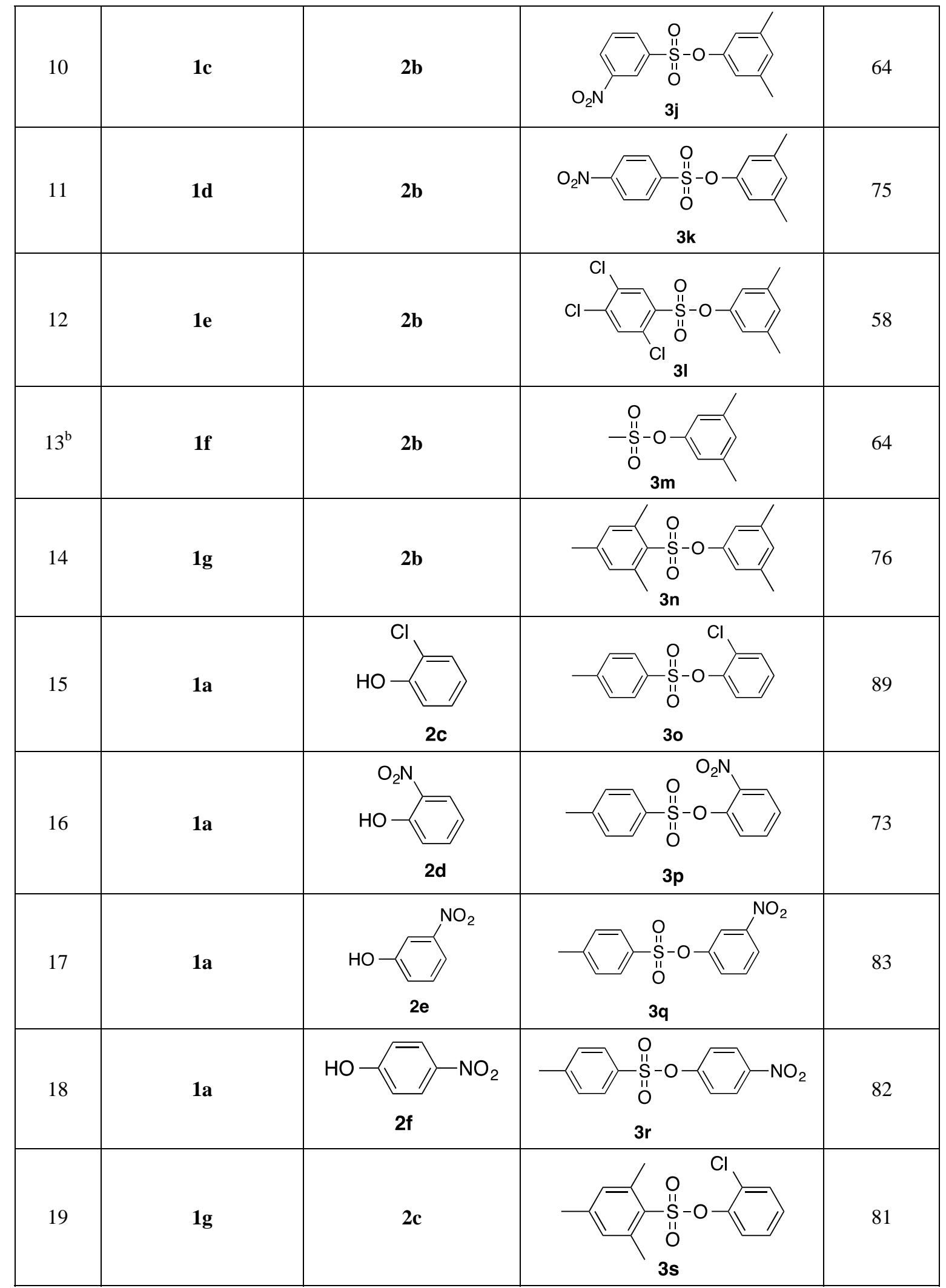


Table 1 continued..

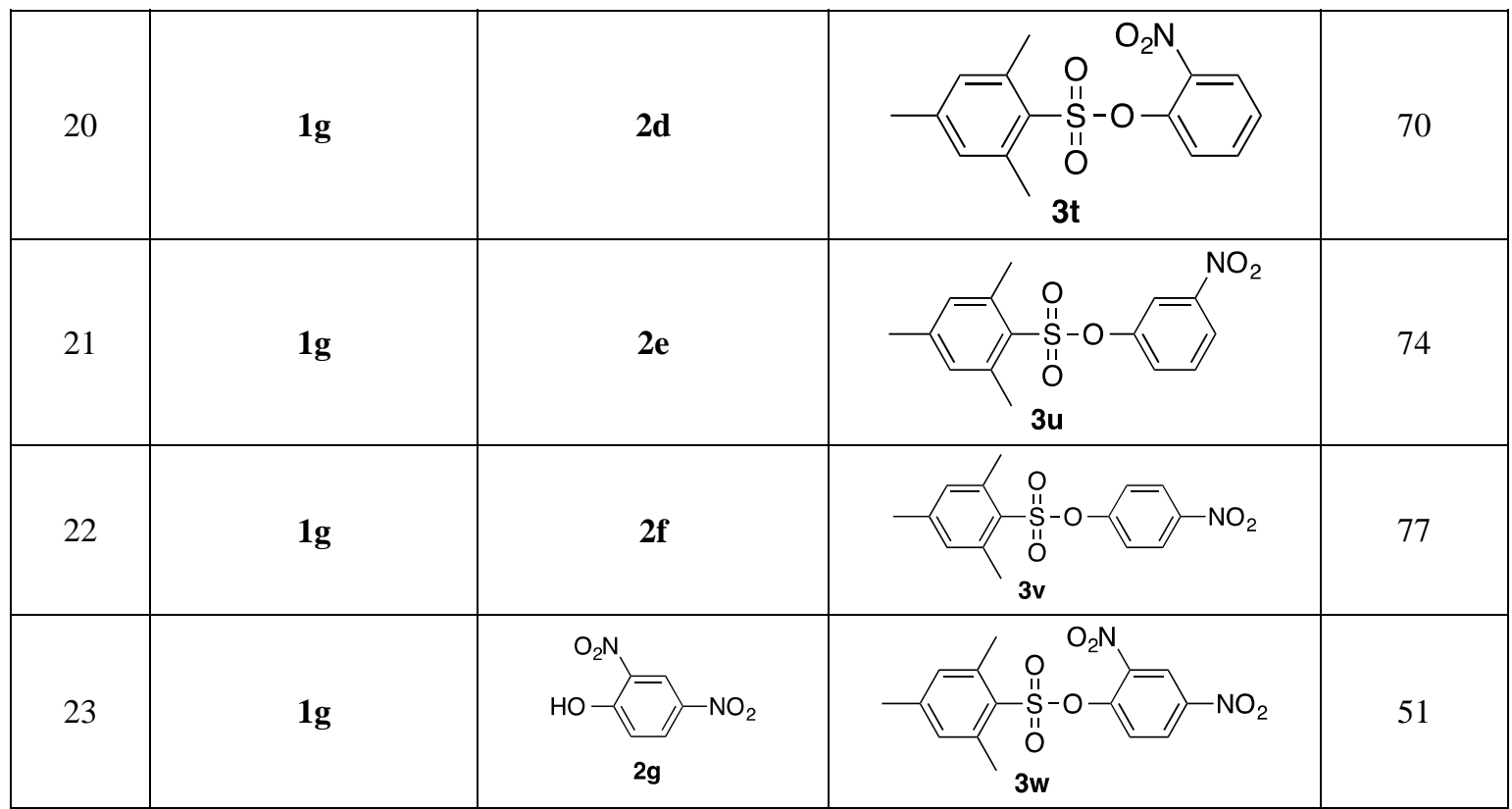

${ }^{a}$ Yields represent isolated yields of pure product after recrystallization (average of two trials). ${ }^{\text {b }}$ Yields represent isolated yields of pure product after column chromatography.

$82 \%$ of products, respectively (substrates $1 \mathrm{a}$ and 1g, entries 1 and 7). A successful reaction with the sterically highly crowded mesitylene sulfonyl chloride $1 \mathrm{~g}$ could be an indication that the reaction condition can adapt to steric constraints in the sulfonyl chloride partner (Table 1, entry 7). Methanesulfonyl chloride was successfully employed to give the sulfonate product 3f in an excellent $97 \%$ yield (entry 6). Sulfonyl chloride derivatives bearing electron-withdrawing groups $\left(-\mathrm{NO}_{2}\right.$ and $\left.-\mathrm{Cl}\right)$ also reacted to give the sulfonate products in fairly good yields (Table 1, entries 2-4, 5 and 8). With the electron-withdrawing group $\left(-\mathrm{NO}_{2}\right)$ in the ortho, meta and para positions, the yields of products were almost comparable. With only one nitro group, a relatively greater yield was obtained with the nitro group in the para position, followed by the ortho position and then the meta position (Table 1, entries $2-4)$. With two electron-withdrawing groups $\left(-\mathrm{NO}_{2}\right)$, in the ortho and para positions, a slightly lower yield of $74 \%$ was obtained (Table 1 , entry 8 ). When a sulfonyl chloride containing three electronwithdrawing groups $(-\mathrm{Cl})$, in the ortho, meta and para positions was used, only a modest yield of $56 \%$ was obtained (Table 1, entry 5).
Using different sulfonyl chloride derivatives, we changed the nucleophilic partner from phenol to 3,5-dimethyl phenol (Table 1, entries 9-14). Compared to the results using phenol as the nucleophilic partner, the yields using 3,5-dimethylphenol with the sulfonyl chlorides $\mathbf{1 a - 1 g}$ are generally lower except in the case of 1e. Thus, 3,5-dimethylphenol reacted with the sulfonyl chloride $\mathbf{1 a}$ to give $\mathbf{3 i}$ in an excellent $90 \%$ yield (Table 1, entry 9). The sulfonyl chlorides with electron-withdrawing groups $\left(-\mathrm{Cl}\right.$ or $\left.-\mathrm{NO}_{2}\right)$ reacted with 3,5-dimethylphenol to give fairly good yields of the sulfonate products 3j-3l (Table 1, entries 10-12). The reaction with methanesulfonyl chloride could only be purified by column chromatography because all attempts to purify the product by recrystallization failed. A reasonably good $64 \%$ yield of the sulfonate product $3 \mathbf{m}$ was obtained when methanesulfonyl chloride reacted with 3,5-dimethlphenol (Table 1, entry 13). The highly sterically congested mesitylene sulfonyl chloride reacted with 3,5-dimethylphenol to give a fairly good yield of $76 \%$ of the sulfonate product 3n (Table 1, entry 14). The results obtained using different sulfonyl chlorides with varying substituents and reacting these with either phenol or 3,5-dimethylphenol do not clearly indicate if 
one particular factor, electronic or steric, is responsible for the difference in product yield. The effects of substituents on the benzene ring of sulfonyl chlorides in the synthesis of sulfonamides from $N$-hydroxybenzotriazole esters of sulfonic acids (TsOBt) have been reported [6]. Mandal et al. reported that electron withdrawing groups increased the yield and reduced the reaction time in the synthesis of sulfonamides from TsOBt [6]. Considering the results together, we can envisage a synergy between electronic and steric factors as being responsible for the different product yields.

Our next target was to investigate the scope of the reaction with respect to the nature of the nucleophilic partner using either tosyl chloride as the electrophilic partner (Table 1, entries 15-18) or mesitylene sulfonyl chloride as the electrophilic partner (Table 1, entries 19-23). Phenol derivatives with electron-withdrawing groups gave yields that could partially be rationalized using the electronic effect of the substituents. The electronic effect of substituents on the phenol derivatives in the reaction with tosyl chloride had previously been reported $[5,18]$. The presence of an electron-withdrawing group on the benzene ring makes the phenol derivative less nucleophilic. The elctron-withdrawing group can influence the nucleophilicity by either inductive effect or resonance. As resonance has a stronger effect than induction in delocalizing the electron density on the nucleophilic center, the chloro-substituted phenol showed relatively greater product yield than the nitro-substituted phenol. Thus 2-chlorophenol reacts with tosyl chloride to give the sulfonate product in $89 \%$ yield (Table 1, entry 15). Compared to 2-chlorophenol, 2-nitrophenol reacted to give a modest yield of $73 \%$ of the sulfonate product 3p (Table 1, entry 16). The electron-withdrawing resonance of the nitro group is limited to the ortho and para positions. The nitro group in the ortho position gave a lower yield of $73 \%$ compared to the $82 \%$ obtained when the nitro group is found in the para position (Table 1, entries 16 and 18). The difference could be explained in terms of the steric hindrance created by the nitro group in the ortho position adjacent to the phenolic $\mathrm{OH}$. The further the nitro group from the phenolic $\mathrm{OH}$, the more nucleophilic the phenol derivative and the higher the yield. This could explain why the yield of the product when the nitro group is in the meta position is $83 \%$ (Table 1 , entry 17) and is greater than when it is in the ortho position but comparable to the product obtained when the nitro group is in the para position. Similar results were obtained using mesitylene sulfonyl chloride as the electrophilic partner, albeit with relatively lower product yields (Table 1, entries 19-23). A similar trend was observed in which the products with electron-withdrawing nitro groups gave lower yields than the product with electronwithdrawing chloro groups. Thus, irrespective of the electronic and steric nature of the phenol derivatives, good to moderate yields were obtained (Table 1, entries 19-23). Even the crowded and relatively weaker nucleophilic 2,4-dinitrophenol $\mathbf{2 g}$ reacted to give an acceptable 51\% considering the strong electron-withdrawing character of the two nitro groups and the steric hindrance.

The mechanism for the nucleophilic substitution reaction is a series of addition-elimination sequence, in which the pyridine serves as both an acid scavenging agent and a nucleophilic acid-base catalyst (Scheme 1).

The sulfonylpyridinium salt, $\mathbf{B}$, is more active than the sulfonyl chloride $\mathbf{A}$ despite significantly higher acidity of $\mathrm{HCl}(\mathrm{pKa}=-7)$ than the pyridinium cation (approximate $\mathrm{pKa}=5.2$ ). This is not surprising because the atom of nitrogen is lacking a lone electron pair conjugated with the sulfonyl, and bears a formal positive charge, which makes the sulfonylpyridinium salt highly electrophilic. With no lone pair of electrons on the nitrogen of the sulfonylpyridinium salt, there is therefore no overlap with $\mathrm{S}=\mathrm{O} \delta+$ orbital and the pyridinium ion is strongly electron withdrawing. The overall mechanism involves two sulfonyl substitution reactions, and because pyridine is acting as a nucleophile in the first nucleophilic substitution but is not consumed in the reaction, it acts as a nucleophilic catalyst. In effect, pyridine serves as both an acid scavenging agent and a nucleophilic acid-base catalyst (Scheme 1).

Nucleophilic aromatic substitution by the additionelimination mechanism $S_{N} A r$ is a very important reaction in organic synthesis [19-24]. The use of sulfonates as electrophilic partners in $\mathrm{S}_{\mathrm{N}} \mathrm{Ar}$ reactions has been reported recently [25, 26]. Using suitable nitrogen-containing and oxygencontaining nucleophiles, two competitive reaction 


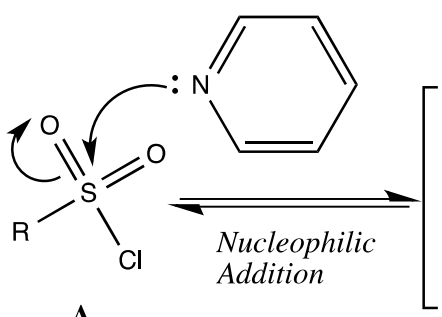

A

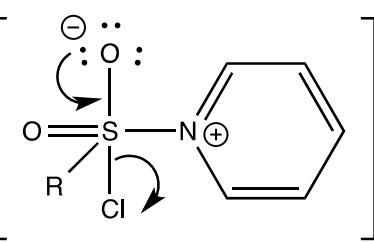<smiles>CC1CCCCC1C(=O)Cl</smiles>

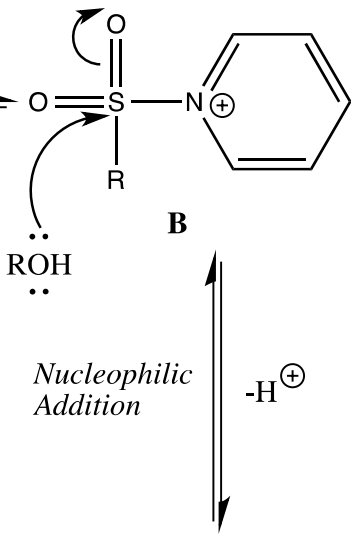<smiles>c1ccncc1</smiles><smiles>[2H]S(=O)(=O)O</smiles><smiles>C1=CC=C1</smiles>

C<smiles>[2H]S(=O)(=O)N(CC)CCO</smiles>

Scheme 1. Proposed mechanism for nucleophilic substitution between phenol and sulfonyl chloride in pyridine.

Table 2. $\mathrm{S}_{\mathrm{N}} \mathrm{Ar}$ reaction of 2,4-dinitrophenyl 4-methylbenzenesulfonate with $\mathbf{5 a}$ and $\mathbf{5} \mathbf{b} .{ }^{\mathrm{a}}$<smiles>Cc1ccc(S(=O)(=O)Oc2ccc([N+](=O)[O-])cc2[N+](=O)[O-])cc1</smiles>

4

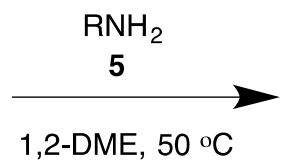

1,2-DME, $50^{\circ} \mathrm{C}$<smiles>Nc1ccc([N+](=O)[O-])cc1[N+](=O)[O-]</smiles>

6<smiles>Cc1ccc(S(=O)(=O)NP)cc1</smiles>

7
$\mathrm{a}: \mathrm{R}=\overbrace{\text { allylamine }}^{\mathrm{NH}_{2}}$
b: $\mathrm{R}=\overbrace{\mathrm{NH}_{2}}$
1-butanamine

\begin{tabular}{|c|c|c|c|c|}
\hline Entry & Tosylate & Amine & Product 6/Yield (\%) & Product 7/Yield (\%) \\
\hline 1 & 4 & $\begin{array}{l}\text { allylamine } \\
\mathbf{5 a}\end{array}$ & $\pi \underbrace{(84 \%)}_{6 \mathbf{a}}$ & $\underset{(11 \%)}{7 a}$ \\
\hline 2 & 4 & $\begin{array}{l}\text { 1-butanamine } \\
\mathbf{5 b}\end{array}$ & $\sqrt{ }$ & $\begin{array}{l}7 b \\
(8 \%)\end{array}$ \\
\hline
\end{tabular}

${ }^{\mathrm{a}}$ The reaction was carried out at $50{ }^{\circ} \mathrm{C}$ in 1,2-DME $(0.1 \mathrm{M})$ under a nitrogen atmosphere using 4 (1.25 mmol) and 5 (5 molar equiv). ${ }^{b}$ Determined by ${ }^{1} \mathrm{HNMR}$ analysis after column chromatography purification. 
pathways (notably, C-O bond-cleavage and S-O bond-cleavage) have been reported [27]. To investigate the application of arylsulfonates as electrophilic partners in $\mathrm{S}_{\mathrm{N}} \mathrm{Ar}$ reactions, we decided to take advantage of the potential high reactivity of 2,4-dinitrophenyl 4-methylbenzenesulfonate towards nucleophiles in $\mathrm{S}_{\mathrm{N}} \mathrm{Ar}$ reactions [28]. With the sulfonate in hand, we designed our initial experiment choosing amines with different nucleophilicity in order to investigate the regioselectivity of $S_{N} A r$ reactions with sulfonates. As a representative example, 6 could be synthesized from 2,4-dinitrophenyl 4-methylbenzenesulfonate 4 and primary amine 5 . We used the general $S_{N} A r$ reaction conditions [29]. Our initial experiment was carried out by heating to $50^{\circ} \mathrm{C}$ in 1,2-DME until all starting materials were consumed as monitored by TLC. The reactions were purified by silica gel column chromatography, and the regiochemistry of the products confirmed by NMR. Gratifyingly, when allylamine $\mathbf{5 a}$ was used as the nucleophilic partner under the standard $\mathrm{S}_{\mathrm{N}} \mathrm{Ar}$ reaction conditions, $N$-allyl-2,4-dinitroaniline $\mathbf{6 a}$ was obtained as the major product in $84 \%$ yield and $N$-allyl-4methylbenzenesulfonamide $\mathbf{7 a}$ as the minor product in $11 \%$ yield (Table 2, entry 1). Using 1butanamine as the nucleophilic partner afforded $81 \%$ of the major product $N$-butyl-2,4-dinitroaniline 6b and $8 \%$ of the minor product N-butyl-4methylbenzenesulfonamide $\mathbf{7 b}$ (Table 2, entry 2). Further studies are underway in our laboratory with the aim of broadening the scope of the $\mathrm{S}_{\mathrm{N}} \mathrm{Ar}$ reactions and optimizing the regioselectivity.

\section{CONCLUSION}

In summary, we have developed a facile synthesis of sulfonate derivatives in which the pure products are obtained without column chromatography. A wide range of sulfonate derivatives was obtained in good to excellent yields by recrystallization of the crude product after initial workup. Noteworthy is the simple separation and purification of the crude product by simple recrystallization that is less time consuming compared to traditional column chromatography. Initial experiments were carried out using the prepared sulfonates as electrophilic substrates in $\mathrm{S}_{\mathrm{N}} \mathrm{Ar}$ reactions with nitrogen-containing nucleophiles. The results obtained have shown these sulfonates as good electrophilic partners for $\mathrm{S}_{\mathrm{N}} \mathrm{Ar}$ reactions. Further experiments are underway to study the regioselectivity of S-O vs. C-O bondcleavage in the $S_{N} A r$ reactions using sulfonates as the electrophiles.

\section{ACKNOWLEDGEMENTS}

We acknowledge the generosity of the Chemistry Department at GVSU through the use of its instrumentation facilities. We also thank the Chemistry Department for providing financial support through the Weldon Fund.

\section{CONFLICT OF INTEREST STATEMENT}

There are no conflicts of interest.

\section{REFERENCES}

1. Chauhan, P., Mahajan, S. and Enders, D. 2014, Chem. Rev., 114, 8807-8864.

2. Kabalka, G. W., Verma, M. and Varma, R. S. 1986, J. Org. Chem., 51, 2386-2388.

3. Musicki, B. and Widlanski, T. S. 1992, Tetrahedron Lett., 32, 1267-1270.

4. Cho, C.-H., Yun, H.-S. and Park, K. 2003, J. Org. Chem., 68, 3017-3025.

5. Zhao, N., Li, Y., Wang, Y. and Wang, J. 2006, J. Sulfur Chem., 27, 427-432.

6. Palakurthy, N. B. and Mandal, B. 2011, Tetrahedron Lett., 52, 7132-7134.

7. Zhang, Y., Lavigne, G. and César, V. 2015, J. Org. Chem., 80, 7666-7673.

8. Sureshan, K. M., Shashidhar, M. S., Praveen, T., Gonnade, R. G. and Bhadbhade, M. M. 2002, Carbohydr. Res., 337, 2399-2410.

9. Miller, S. C. 2010, J. Org. Chem., 75, 46324635.

10. Gelman, D. and Buchwald, S. L. 2003, Angew. Chem. Int. Ed., 42, 5993-5996.

11. Choy, P. Y., Chow, W. K., So, C. M., Lau, C. P. and Kwong, F. Y. 2010, Chem. Eur. J., 16, 9982-9985.

12. (a) Ngassa, F. N., Gomez, J. M., Haines, B. E., Ostach, M. J., Hector, J. W., Hoogenboom, L. J. and Page, C. E. 2010, Tetrahedron, 66, 7919-7926. (b) R'kyek, O., Halland, N., Lindenschmidt, A., Alonso, J., Lindemann, P., Urmann, M. and Nazaré, M. 2010, Chem. Eur. J., 16, 9986-9989. (c) Quan, Z. J., Jing, F. Q., Zhang, Z., Da, Y. X. and Wang, X. C. 2013, Eur. J. Org. Chem., 2013, 7175-7183; 
(d) Delbianco, M., Lamarque, L. and Parker, D. 2014, Org. Biomol. Chem., 40, 8061-8071; (e) Moore, J. N., Laskay, N. M., Duque, K. S., Kelley, S. P., Rogers, R. D. and Shaughnessy, K. H. 2015, J. Organomet. Chem., 777, 16-24.

13. Chow, W. K., So, C. M., Lau, C. P. and Kwong, F. Y. 2014, Org. Chem. Front., 1, 464-467.

14. Teasdale, A., Delaney, E. J., Eyley, S. C., Jacq, K., Taylor-Worth, K., Lipczynski, A., Hoffmann, W., Reif, V., Elder, D. P., Facchine, K. L., Golec, S., Oestrich, R. S., Sandra, P. and David, F. 2010, Org. Process Res. Dev., 14, 999-1007.

15. (a) Jain, P., Yi, S. and Flaherty, P. T. 2013, J. Heterocyclic Chem., 50, E166-E173; (b) Li, Q., Zhang, L. M., Bao, J. J., Li, H. X., Xie, J. B. and Lang, J. P. 2014, Appl. Organometal. Chem., 28, 861-867; (c) Mondal, K. and Pan, S. C. 2015, Eur. J. Org. Chem., 2015, 21292132; (d) Wang, X. X., Xu, B. B., Song, W. T., Sun, K. X. and Lu, J. M. 2015, Org. Biomol. Chem., 13, 4925-4930.

16. (a) Li, B. J., Yu, D. G., Sun, C. L. and Shi, Z. 2011, J. Chem. Eur. J., 17, 1728-1759;

(b) Pompeo, M., Froese, R. D. J., Hadei, N. and Organ, M. G. 2012, Angew. Chem. Int. Ed., 51, 11354-11357; (c) Nxumalo, W. and Dinsmore, A. S. 2013, Afr. J. Chem., 66, 42-46.

17. (a) Li, X. F., Paoloni, F. P. V., Weiber, E. A., Jiang, Z. H. and Jannasch, P. 2012, Macromolecules, 45, 1447-1459; (b) Baqi, Y. and Mueller, C. E. 2012, Molecules, 17, 2599-2615; (c) Quan, Z., Jing, F., Zhang, Z., Da, Y. and Wang, X. 2013, Chin. J. Chem., 31, 1495-1502; (d) Dabiri, M., Shariatipour, M., Kazemi Movahed, S. and Bashiribod, S. 2014, RSC Adv., 4, 39428-39434.

18. Meshram, G. A. and Patil, V. D. 2009, Tetrahedron Lett., 50, 1117-1121.
19. Makosza, M. 2010, Chem. Soc. Rev., 39, 2855-2868.

20. Senger, N. A., Bo, B., Cheng, Q., Keeffe, J. R., Gronert, S. and Wu, W. 2012, J. Org. Chem., 77, 9535-9540.

21. Petersen, T. P., Larsen, A. F., Ritzén, A. and Ulven, T. 2013, J. Org. Chem., 78, 41904195.

22. Scales, S., Johnson, S., Hu, Q., Do, Q.-Q., Richardson, P., Wang, F., Braganza, J., Ren, S., Wan, Y., Zheng, B., Faizi, D. and McAlpine, I. 2013, Org. Lett., 15, 2156-2159.

23. Gronert, S., Garver, J. M., Nichols, C. M., Worker, B. B. and Bierbaum, V. M. 2014, J. Org. Chem., 79, 11020-11028.

24. Fier, P. S. and Hartwig, J. F. 2014, J. Am. Chem. Soc., 136, 10139-10147.

25. Chassaing, S., Specklin, S., Weibel, J.-M. and Pale, P. 2012, Tetrahedron, 68, 7245-7273.

26. Imazaki, Y., Shirakawa, E., Ueno, R. and Hayashi, T. 2012, J. Am. Chem. Soc., 134, 14760-14763.

27. (a) Pregel, M. J., Dunn, E. J. and Buncel, E. 1991, J. Am. Chem. Soc., 113, 3545-3550; (b) Um, I. H., Hong, J. Y., Kim, J. J., Chae, O. M. and Bae, S. K. 2003, J. Org. Chem., 68, 5180-5185; (c) Um, I. H., Chun, S. M., Chae, O. M., Fujio, M. and Tsuno, Y. 2004, J. Org. Chem., 69, 3166-3172; (d) Quan, Z., Jing, F., Zhang, Z., Da, Y. and Wang, X. 2013, Chin J. Chem., 31, 1495-1502; (e) Um, I. H., Kang, J. S., Shin, Y. H. and Buncel, E. 2013, J. Org. Chem., 78, 490-497.

28. Cooley, T. A., Riley, S., Biros, S. M., Staples, R. J. and Ngassa, F. N. 2015, Acta Cryst., E71, 1085-1088.

29. Lakshman, M. K., Ngassa, F. N., Keeler, J. C., Dinh, Y. Q. V., Hilmer, J. H. and Russon, L. M. 2000, Org. Lett., 2, 927-930. 